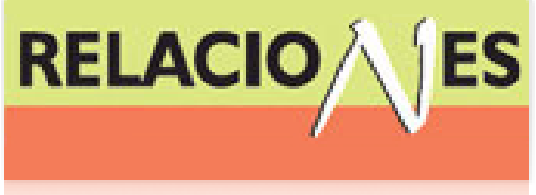

Relaciones. Estudios de historia y sociedad ISSN: 0185-3929

relacion@colmich.edu.mx

El Colegio de Michoacán, A.C

México

M. Toledo, Víctor

El metabolismo social: una nueva teoría socioecológica

Relaciones. Estudios de historia y sociedad, vol. XXXIV, núm. 136, 2013, pp. 41-71

El Colegio de Michoacán, A.C

Zamora, México

Disponible en: http://www.redalyc.org/articulo.oa?id=13729711004

- Cómo citar el artículo

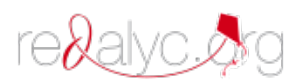

- Número completo

- Más información del artículo

Página de la revista en redalyc.org

Sistema de Información Científica

Red de Revistas Científicas de América Latina, el Caribe, España y Portugal Proyecto académico sin fines de lucro, desarrollado bajo la iniciativa de acceso abierto 


\title{
El metabolismo social: una nueva teoría socioecológica
}

\author{
Víctor M. Toledo*
}

CIECO-UNAM

A la memoria de

Alfred Schmidt (1931-2012)

Ante la necesidad de construir marcos conceptuales que permitan realizar análisis congruentes sobre las relaciones entre los procesos naturales y los procesos sociales desde una perspectiva holística o integradora, el artículo lleva a cabo una narrativa de la teoría del metabolismo social, uno de los instrumentos propuestos en la última década para comprender la compleja realidad actual. Para ello se hace una breve revisión de los orígenes del concepto y una síntesis de sus principales significados. El ensayo termina llamando la atención sobre el potencial teórico del concepto, no obstante que existen diferentes interpretaciones y versiones del mismo.

(Metabolismo social, socioecología, naturaleza y sociedad, Marx, Alfred Schmidt) INTRODUCCIÓN

n la primavera de 1973 , este autor descubrió en una librería
de la ciudad de Cambridge, Massachussets, EU, la versión
inglesa de la obra de Alfred Schmidt, El Concepto de Natura-
leza en Marx, en una edición de 1971 . El libro era la traducción de
la tesis de doctorado de ese filósofo de la llamada Escuela de
Frankfurt publicada en alemán en forma de libro en 1962. La obra,
cuya primera edición en español apareció hasta 1976, resultó a la

*vtoledo@cieco.unam.mx 
larga seminal en la evolución intelectual de quien esto escribe, especialmente en la decantación del metabolismo social, un concepto que hoy es quizás el instrumento teórico más poderoso para analizar de manera conjunta las relaciones entre los procesos naturales y los procesos sociales (Toledo 2011). A través de los ańos, el libro se tradujo a dieciocho idiomas, y se convirtió en lectura obligada para quienes se interesan en la articulación entre marxismo y ecología. Alfred Schmidt murió apenas el 28 de agosto de 2012 en Frankfurt/Main a la edad de ochenta y un años (Gandler 2012). Por todo lo anterior, este artículo es un modesto homenaje a la memoria de ese autor.

\section{EL PROBLEMA SEMINAL}

La circunstancia central del mundo contemporáneo es la de una creciente preocupación por el futuro, pues la sociedad se encuentra inmersa dentro de un "gigantesco experimento sin control" (Mc Neill 2000), provocado por ella misma, donde los procesos naturales y los sociales se articulan de una manera sin precedente, generando nuevas dinámicas y sinergias impredecibles y sorpresivas que amenazan a la especie humana, al equilibrio planetario y a la vida toda. Se trata de una crisis de la civilización moderna o industrial, cuya solución requiere de nuevos paradigmas en todos los campos de la realidad, la que hoy ha quedado convertida en un complejo socionatural o naturosocial (Toledo 2012). Frente a la situación anterior, la ciencia en su conjunto está obligada a conocer el pasado para aprender de él (obtener lecciones); a adoptar una rigurosa perspectiva histórica que permita la cabal comprensión de las situaciones del presente, lo cual implica: a) El desarrollo de un marco conceptual integrador (interdisciplinario) de carácter socioecológico, capaz de orquestar la investigación sobre las relaciones entre la sociedad y la naturaleza; y b) que sea funcional (útil) para el análisis de esas relaciones, es decir, a través de la historia (tiempo) y a diferentes escalas (espacio). Las siguientes secciones están dedicadas a realizar una narrativa de la teoría del metabolismo social, uno de los instrumentos propuestos en la última década para comprender la compleja realidad actual. 


\section{DARWIN y MARX EN LONDRES}

El siglo XIX fue la era del Imperio inglés y Londres se convirtió no solamente en la ciudad más grande del mundo (pasó en cien años de un millón a 6.7 millones de habitantes), sino en la capital mundial de las finanzas, el comercio, la política y la creación intelectual. Ahí vivieron y trabajaron innumerables estudiosos e investigadores de la intelectualidad más avanzada de la época, incluyendo a dos de los grandes pensadores del siglo: Charles Darwin (1809-1892) y Karl Marx (1818-1883). El primero estableció su hogar definitivo en un pueblo cercano a la capital inglesa en 1842 tras su largo viaje de exploración por el sur del mundo. El segundo arribó a Londres siete años después tras un periplo que lo llevó por varias ciudades europeas, y ahí permaneció hasta su muerte. Aunque ambos se encontraban a menos de dieciséis millas de distancia, jamás se conocieron personalmente. Sólo hubo una breve correspondencia epistolar iniciada por Marx, quien fue admirador de la obra de Darwin, a tal punto que intentó dedicarle el tomo dos de El capital.Tras la aparición en noviembre de 1859 de la obra largamente esperada, El origen de las especies, cuya edición se agotó en solamente un día, F. Engels escribió a Marx: "Darwin, a quien por cierto estoy justamente leyendo ahora, es absolutamente espléndido. Su libro ha logrado demoler una cierta teleología que desde hace tiempo se mantenía”. En respuesta, Marx escribió a Engels el 19 de diciembre de 1860: "Este es el libro que contiene las bases histórico naturales de nuestra concepción".

Aunque las conexiones teóricas e intelectuales de Darwin y Marx han sido abordadas con cierta frecuencia por los historiadores de la ciencia (e.g. Gerratana 1973; Colp 1974) o los filósofos marxistas (Foster 2000), debemos a A. Schmidt (1976) lo que ha resultado una revelación crucial. Marx, no solamente había leído extensamente a los naturalistas de su época, también había abrevado de un autor clave, el holandés Jacob Moleschott (1822-1893), reconocido ampliamente en los círculos de las ciencias naturales de Europa, quien escribió varios libros entre los que destaca Der Kreislauf des Lebens (El ciclo de la vida, 1852), un verdadero tratado de ecología decimo- 
nónico. De esa lectura Marx derivó el concepto clave que le permitió construir su teoría crítica sobre el capitalismo: el de Stoffwechsel, que significa intercambio orgánico o metabolismo. Marx utilizó el término (Stoffwechsel) en borradores escritos al final de la década de 1850 y en el volumen I de El capital publicado en Hamburgo en 1867 (véase también Martinez-Alier con Shlüpmann 1987, 220-226).

Marx utilizó ese concepto en dos principales sentidos: como una analogía o metáfora biológica para ilustrar la circulación de las mercancías, y de manera más general como un "intercambio entre hombre y tierra", o un "intercambio entre sociedad y naturaleza" (Martinez-Alier 2004). Schmidt (1976) de nuevo dedicó buena parte de su libro para realizar una detallada interpretación del uso del concepto de metabolismo y reflexionar sobre sus implicaciones sociales, históricas, ecológicas y cósmicas, sintetizadas en una premisa dialéctica clave: "Marx concibió el trabajo como un proceso de progresiva humanización de la naturaleza, un acto que coincide con la gradual naturalización del hombre" $(1976,81)$. La naturaleza es entonces concebida por Marx como el sustrato material del trabajo, como "la primera fuente de todos los medios y objetos de trabajo" (Marx, Crítica del programa Gota). "Todo acto de dar forma a una sustancia natural debe obedecer a la legalidad peculiar de la materia", afirma Schmidt $(1976,84)$, por ello "el hombre sólo puede proceder en su producción como la naturaleza misma, es decir, sólo puede cambiar las formas de la sustancia” (Marx, El capital, tomo I, 47).

La conexión que hace Marx de su teoría económica con los procesos naturales, surge de una distinción clave entre valor de uso y valor de cambio:

Como creador de valores de uso, como trabajo útil, el trabajo es por lo tanto una de las condiciones de existencia del hombre independiente de las formas sociales, constituye una necesidad natural eterna para mediar el metabolismo entre el hombre y la naturaleza, y por lo tanto la vida humana (Marx, El capital, tomo I, 47).

Por ello Marx les llama, respectivamente, la forma natural prosaica, concreta y particular del trabajo; y la forma supranatural, abs- 
tracta y general del trabajo. Esta distinción permite a su vez hacer una diferencia tácita entre intercambio ecológico e intercambio económico (Toledo 1981), lo que resulta crucial en la construcción de una teoría económico-ecológica.

De todo lo anterior se deriva una afirmación, que se adelanta como un vaticinio epistemológico a las actuales propuestas interdisciplinarias: "Las ciencias de la naturaleza subsumirán igualmente en el futuro a las ciencias del hombre, así como las ciencias del hombre a las ciencias de la naturaleza; habrá una sola ciencia" (Marx, Nationalokonomie und Philosophie).

La importancia del concepto de metabolismo en el pensamiento de Marx, quedó certificado por la carta enviada a su esposa el 21 de junio de 1856: "no es el amor por el hombre de Feuerbach, ni por el metabolismo de Moleschot, ni por el proletariado, sino el amor por la mujer querida, en este caso por ti, lo que hace que un hombre vuelva a ser hombre" (véase Annali del Instituto Giangiacomo Feltrinelli, Milán, 1959).

EL REDESCUBIRIMIENTO DEL CONCEPTO DE METABOLISMO SOCIAL

El concepto permaneció en estado latente durante décadas, hasta que a finales de la década de los sesenta del siglo pasado algunos economistas como K. Boulding y R. Ayres lo (re)inventaron, sin saber de su origen, y especialmente cuando Marina Fisher-Kowalski publicó en 1997 un capítulo en el libro Handbook of Environmental Sociology (Redclift y Woodgate 1997), donde lo presentó como un naciente "concepto estelar" utilizado para realizar "análisis de flujos de materiales". Ese y otros artículos de la economista austriaca Fisher-Kowalski trazaron con sumo detalle la historia del concepto y, sobretodo, mostraron sus aplicaciones y enormes potencialidades (Fisher-Kowalski 1998; Fisher-Kowalski y Huttler 1999). Resulta curioso que ninguno de los ensayos sobre marxismo y ecología aparecidos dos décadas atrás hayan registrado el carácter seminal del concepto (Moscovici 1969; Skirbek 1974; Lefevre 1978). Aún la detallada compilación de Parsons (1977) sobre las "ideas ecológicas" de Marx y Engels pasó por alto ese concepto esencial. 
La mayoría de los análisis que utilizan este concepto se han concentrado en cuantificar los flujos de energía y de materiales. El número de estudios que utilizan este concepto se ha incrementado de manera notable, abordando aspectos tales como la salud humana, el desarrollo social y el crecimiento económico (e.g. Ayres y Simonis 1994; Opschoor 1997). En la actualidad se dispone ya de métodos, índices y fuentes de información estadística para calcular con detalle los flujos de materia y energía a escala nacional, de tal suerte que se ha logrado cuantificar el metabolismo energético y/o material de varios países (Matthews et al., 2000; Haberl 2002) e incluso sus cambios a través del tiempo (e.g. Krausmann y Haberl 2002). La mayoría ofrecen diagnósticos contemporáneos, y casi sin excepción se circunscriben al análisis de los flujos de energía y materia y su correlación con ciertos parámetros económicos (Haberl 2001a y 2001b; Opschoor 1995). Hoy existen "perfiles metabólicos" de una treintena de países, incluyendo los de la Comunidad Europea y naciones de Latinoamérica (Chile, Ecuador, México, Perú y Venezuela) y de Asia (China, Laos, Filipinas y Tailandia) (Eisenmenger y Giljum 2007; Russi et al., 2006).

En años recientes, los estudios a esta escala han comenzado a realizar análisis comparativos entre países (Eisenmerger y Giljum 2007; Russi et al., 2006; Muradian y Giljum 2007; Muradian et al., 2002), a intentar diagnósticos históricos (Carpintero 1995; Krausmann y Haberl 2002 para los casos de Espańa y Austria respectivamente) y a evaluar los impactos sobre los recursos naturales utilizando indicadores como la huella ecológica o la apropiación humana de la producción primaria neta (AHPPN) (Krausmann y Erb 2005). En todos los casos, el uso del concepto de metabolismo social se reduce a los simples cálculos de entradas (apropiación), salidas (excreción), importaciones y exportaciones, dejando fuera de sus análisis tanto las complejas configuraciones del resto del proceso metabólico (lo que encierra la "caja negra", la condición a la que quedan reducidas las naciones), como las dimensiones no materiales o intangibles del metabolismo (véase más adelante).

En el otro extremo existen escasos, aunque interesantes, análisis del metabolismo local (rural o agrario) centrados en el estudio de comunidades, municipios y regiones rurales y en el proceso particu- 
lar de apropiación. Estos estudios analizan con sumo detalle las relaciones de las comunidades humanas estudiadas en su relación tanto con los recursos naturales locales como con los sectores mercantiles con quienes realizan transacciones (intercambios mercantiles), es decir, revelan de manera integrada las articulaciones que existen entre los intercambios ecológicos y los intercambios económicos en territorios concretos. Aunque utilizando diferentes metodologías, estos estudios de escala local o microrregional ofrecen avances en la comprensión del proceso metabólico tanto en su condición contemporánea (Singh et al., 2001; Grau-Satorras 2010; García-Frapolli et al., 2008; Cordón y Toledo 2008; Ortiz y Masera 2008), como en una perspectiva histórica (González de Molina y Guzmán-Casado 2005 y 2008; Tello et al., 2008; Krausmann 2002 y 2004).

\section{¿QUÉ ES EL METABOLISMOS SOCIAL?: LO MATERIAL O TANGIBLE}

El metabolismo entre la naturaleza y la sociedad contiene dos dimensiones o esferas: una material, visible o tangible y otra inmaterial, invisible o intangible. Revisemos la primera. El metabolismo social comienza cuando los seres humanos socialmente agrupados se apropian materiales y energías de la naturaleza (input) y finaliza cuando depositan desechos, emanaciones o residuos en los espacios naturales (output). Pero entre estos dos fenómenos ocurren además procesos en las "entrañas" de la sociedad por medio de los cuales las energías y materiales apropiados circulan, se transforman y terminan consumiéndose (figura 1). Por lo anterior en el proceso general del metabolismo social existen tres tipos de flujos de energía y materiales: los flujos de entrada, los flujos interiores y los flujos de salida. El proceso metabólico se ve entonces representado por cinco fenómenos que son teórica y prácticamente distinguibles: la apropiación (A), la transformación (T), la circulación (C), el consumo (Co) y la excreción (E).

El acto de la apropiación (A) constituye, en sentido estricto, la forma primaria de intercambio entre la sociedad humana y la naturaleza. Mediante A, la sociedad se nutre de todos aquellos materiales, energías, agua y servicios que los seres humanos y sus artefactos requieren como individuos biológicos (energía endosomática) y 
Figura I. El proceso general del metabolismo (de materia y energía) entre sociedad y naturaleza. Véase texto.

\section{El metabolismo social}

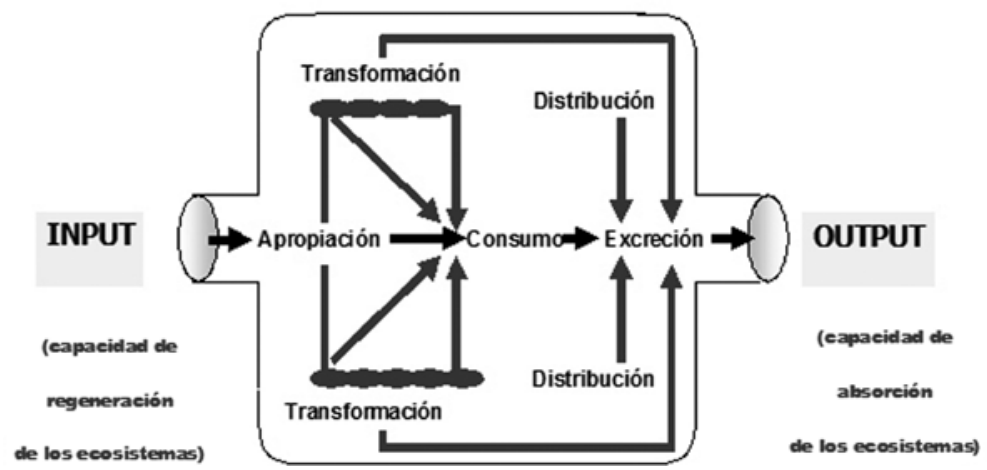

Fuente: González de Molina y Toledo, 2011.

como conjunto social (energía exosomática) para mantenerse y reproducirse. Este proceso lo realiza siempre una unidad de apropiación, la cual puede ser una empresa (estatal o privada), una cooperativa, una familia, una comunidad, o un solo individuo (por ejemplo un captador de energía solar).

El proceso de transformación (T) implica todos aquellos cambios producidos sobre los productos extraídos de la naturaleza, los cuales ya no son consumidos en su forma original. En sus formas más simples, T incluye las modalidades más elementales de preparación de alimentos (por ejemplo, el cocimiento de elementos vegetales o animales por medio del fuego), y en sus modalidades más elaboradas, la transformación de la materia (metalurgia, industria nuclear, biotecnología, petroquímica, nanotecnología, etcétera). A lo largo del tiempo, T se ha ido volviendo gradualmente una actividad más compleja conforme el proceso se ha vuelto menos intensivo en trabajo y más intensivo en el empleo de energías. Pasándose de la artesanía a la manufactura y a la fabricación de productos cada vez más elaborados. Las cadenas o secuencias de transformaciones hacen muchas veces indistinguibles las materias primas, originalmente obtenidas de la naturaleza. 
FIGURA 2. El acto de apropiación en las sociedades actuales, lo realiza el conjunto de unidades de apropiación (P). Los materiales y energías apropiados son después circulados (Cir) por diferentes vías, en su caso transformados (Tr), y finalmente consumidos por la industria y las ciudades. Todos estos procesos generan, a su vez, un flujo de desechos hacia la naturaleza (excreción). Además de sus relaciones metabólicas con la naturaleza (input y output), las sociedades también importan y exportan bienes con otras sociedades.

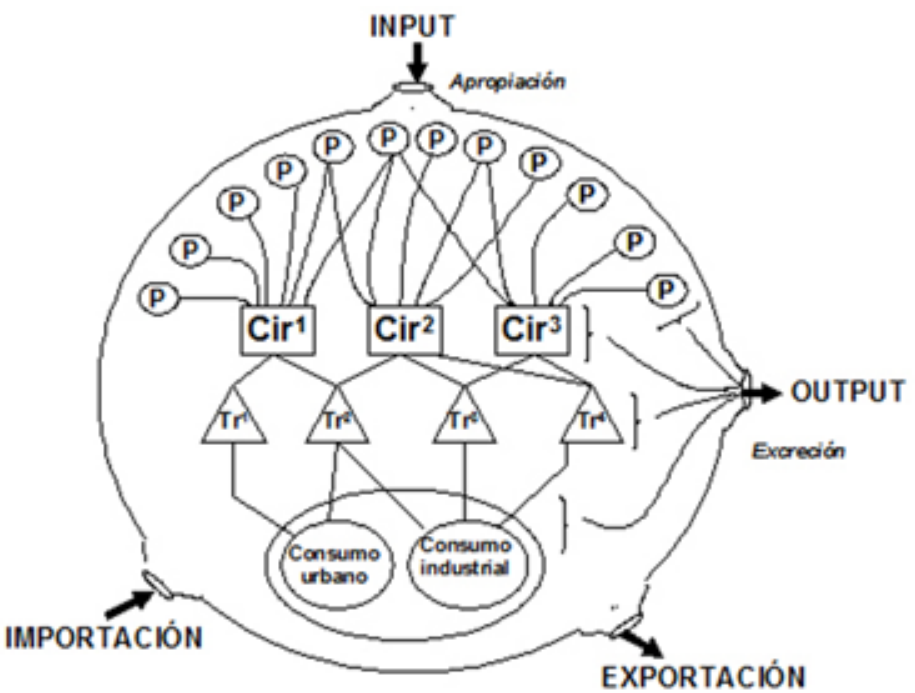

Fuente: González de Molina y Toledo, 2011.

El proceso de circulación (C) aparece en el momento en el que las unidades de apropiación dejan de consumir todo lo que producen y de producir todo lo que consumen. Con ello se inaugura, en sentido estricto, el fenómeno del intercambio económico (Toledo 1981). Los elementos extraídos de la naturaleza comienzan entonces a circular, transformados o no, y en el devenir de la historia se incrementan no sólo los volúmenes de lo que circula, sino las distancias que recorren antes de ser consumidos. Los cambios en los patrones de comunicación territorial logrados a través de formas cada vez más eficientes de transporte (humano, animal, fluvial, marino, aéreo, etcétera) fueron amplificando su radio de distribución, 
hasta llegar al presente donde lo que se circula se hace de manera cada vez más rápida. La magnitud de $\mathrm{C}$ ha ido evolucionando desde la asignación (o el intercambio) no mercantil ni monetario hasta el intercambio mediado por el dinero, la propiedad privada y los mercados. El resultado ha sido una intrincada red de intercambios, íntimamente ligada con las transformaciones, en la que queda difuminada la antigua relación, directa y casi inmediata, entre apropiación y consumo.

En el proceso metabólico del consumo (Co) se ve envuelta toda la sociedad, independientemente de su posición en la cadena metabólica. Este proceso puede ser entendido a partir de la relación que existe entre las necesidades del ser humano, social e históricamente determinados, y los satisfactores proporcionados por medio de los tres primeros procesos $(\mathrm{A}+\mathrm{T}+\mathrm{C})$. No obstante, en muchas sociedades (sobre todo en sociedades de base energética orgánica) el nivel de consumo ha determinado el esfuerzo de A, T, C (sociedades agrarias, por ejemplo) pero especialmente en el actual funcionamiento del metabolismo industrial donde el consumo constituye un poderoso factor de demanda que incentiva y en alguna medida subordina a los demás procesos metabólicos.

De nuevo, en el proceso de excreción (E), que es el acto por el cual la sociedad humana arroja materiales y energía hacia la naturaleza (incluyendo basuras, emanaciones, gases, substancias y calor), también se ve envuelta toda la sociedad y todos los procesos metabólicos. Las dos cuestiones básicas que hay que considerar aquí son: la calidad de los residuos (si son reciclables o no por la naturaleza) y su cantidad (si sobrepasa o no la capacidad natural de reciclaje). Lo anterior además del calor que se genera en toda actividad humana, y que es una respuesta física a toda transformación o movimiento.

¿QUÉ ES EL METABOLISMO SOCIAL? LO INMATERIAL O INTANGIBLE

Los seres humanos, agrupados en sociedad, no sólo comen, beben, sudan, crecen, fornican, excretan y mueren. Tampoco están dedicados solamente a construir estructuras o a elaborar utensilios, instrumentos, armas, mecanismos o máquinas. También sueñan, 
imaginan, creen, conocen, inventan signos y lenguajes para comunicarse, establecen relaciones entre ellos, producen reglas, normas y leyes, diseñan tecnologías, hacen transacciones y construyen instituciones con diferentes fines y en distintas escalas. Y es esta parte intangible de la sociedad la que opera como un armazón para los procesos materiales del metabolismo.

Casi sin excepción, el proceso general de metabolismo ha sido abordado como un fenómeno meramente material (y ello explica porque sus principales analistas han sido por lo común economistas de la nueva corriente de la economía ecológica). Sin embargo, un abordaje sociológico completo obliga a considerar aquellas instancias y mecanismos de carácter no material con los cuales y dentro de los cuales el metabolismo tiene lugar. Desde las sociedades tecnológicamente más simples el proceso metabólico material siempre ha ocurrido, ha estado embebido, dentro de determinadas relaciones sociales, es decir, siempre ha estado condicionado por diversos tipos de instituciones, formas de conocimiento, cosmovisiones, reglas, normas y acuerdos, saberes tecnológicos, modos de comunicación y de gobierno y formas de propiedad.

Los cinco procesos metabólicos se articulan de manera específica, particular y estable a lo largo del tiempo, lo que permite hablar de formas específicas de articulación entre ellos y con la naturaleza. Y son las instituciones, que expresan relaciones estrictamente sociales como la familia, el mercado, las reglas de acceso a los recursos, el poder político, la fiscalidad, el parentesco, el apoyo recíproco, etcétera, junto con otras dimensiones igualmente intangibles como las que hemos señalado, las que suelen organizar socialmente esa articulación de los procesos metabólicos,

Mientras que los primeros procesos operan como la "parte dura" o visible de las sociedades humanas, como su blindaje material y energético; las instituciones, y sus consiguientes sistemas simbólicos, reglas jurídicas y/o sociales funcionan como la "parte blanda" invisible e inmaterial. Por lo anterior, resulta pertinente afirmar que todo metabolismo social tiene un hardware y un software, los cuales se determinan recíprocamente a lo largo de la historia en procesos que hoy resultan aun incomprensibles y que es necesario descubrir y analizar. 
Figura 3. La estructura metabólica de la sociedad aparece como la integración de dos cuerpos poliédricos (uno contenido en el otro): el formado por los cinco procesos materiales (números) y el que conforma el conjunto de dimensiones intangibles (letras). El reto es encontrar las reglas que determinan las sinergias que se dan dentro y entre ambas dimensiones, más su interacción con el universo natural.

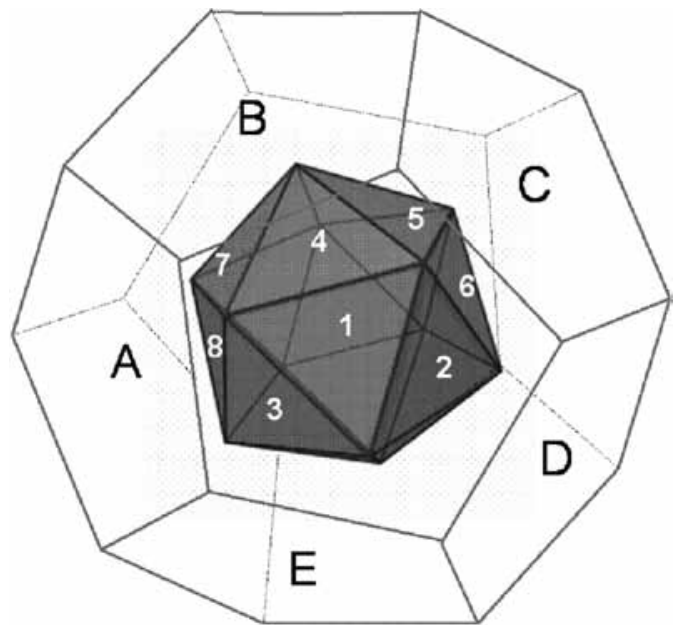

Fuente: González de Molina y Toledo, 2011.

En cada sociedad dada existe, por lo tanto, una articulación específica de los cinco procesos metabólicos, y una constitución específica de las relaciones sociales que configuran cada uno de ellos, que tienden a la reproducción, a la continuidad en el tiempo, al mostrar cierto consenso social a la hora de satisfacer las necesidades básicas. No obstante, creemos que se pueden singularizar, sin afán ontológico alguno, formas más o menos estables de configuración y articulación de los cinco procesos metabólicos y su contraparte simbólica o institucional, que podemos designar de manera tentativa, como sistema social (social stages) o como configuraciones societarias (para detalles véase González de Molina y Toledo 2011).

Una versión completa del metabolismo social, como modelo o marco conceptual para el estudio de las relaciones entre las diferentes sociedades y la naturaleza y sus transformaciones a lo largo 
del tiempo, cristaliza entonces en una estructura poliédrica formada de dos partes (una material, la otra intangible) indisolublemente ensambladas, totalidad que a su vez mantiene relaciones recíprocas, dinámicas y complejas con el mundo de la naturaleza y sus procesos (figura 3). Dentro de esta estructura la porción material o visible opera como el contenido y la parte inmaterial o invisible como la contenedora. Mientras que la primera está representada por procesos materiales, identificables y cuantificables, la segunda se encuentra formada por dimensiones (cognitivas, simbólicas, institucionales, jurídicas, tecnológicas, etcétera). Tocará a los investigadores dilucidar cómo esas dimensiones afectan y determinan a esos procesos, y cómo ese conjunto de determinaciones, de alta complejidad, generan transformaciones a lo largo del tiempo y cuáles son las reglas que operan durante tales transformaciones. Esa estructura conforma, entonces, la unidad-totalidad que se transforma a lo largo del tiempo, pero que también se colapsa o se desorganiza, se reconstruye o se osifica, se hace estacionaria o inestable.

Llevado a su expresión concreta, el modelo abstracto del metabolismo social puede analizarse en su totalidad como proceso, o bien dedicarse a explorar fracciones o porciones del mismo; esto es, puede adoptar una dimensión totalizadora o por el contrario focalizada a ciertas porciones de ese proceso. Lo anterior define la dimensión del análisis. De manera general se pueden identificar tres "campos" de estudio del metabolismo social: agrario o rural, urbano e industrial, cada uno de los cuales se centra más en uno o más de los procesos que forman la cadena metabólica (figura 4).

El proceso general del metabolismo, en tanto que ocupa una posición en el espacio, puede analizarse también a diferentes escalas. La estrechez o amplitud espacial del enfoque definida y/o decidida por el analista revela el carácter multiescalar del estudio del metabolismo social. Grosso modo, se pueden identificar hasta seis categorías definidas por la escala: la de unidad de apropiación/producción, la de comunidad, la microrregional (e.g. municipios o condados), la regional (e.g. cuencas hidrológicas), la nacional, la internacional y la global o de especie. 
Figura 4. Matriz de relaciones entre los tres principales "campos" del proceso general del metabolismo social y los cinco procesos metabólicos.

Procesos metabólicos

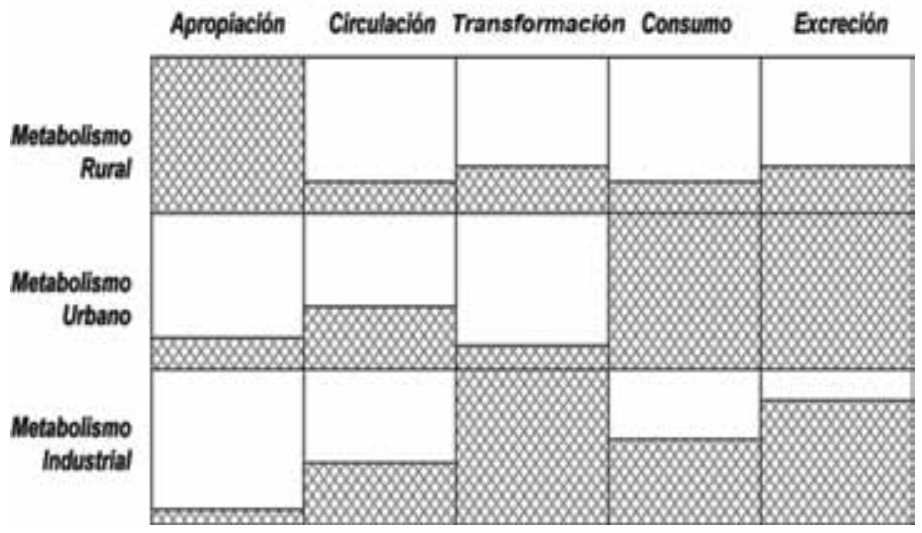

Fuente: González de Molina y Toledo, 2011.

De manera similar, el metabolismo social puede abordarse en diferentes escenarios temporales, identificados por los periodos abordados, cuando se adopta una perspectiva histórica. En este caso es posible reconocer situaciones por la magnitud o la escala del tiempo: ańos, décadas, siglos y milenios. Después de todo, el metabolismo social existe desde la aparición de la especie humana, hace unos 200,000 ańos.

Se arriba entonces a una matriz formada de tres variables, la $d i$ mensión, la escala y el tiempo, que enmarca todo análisis del proceso metabólico (figura 5). Este marco tridimensional pone de manifiesto, además, la estrecha relación que existe entre la dimensión, la escala y el tiempo, en tanto que son tres aspectos en permanente reciprocidad. Por ejemplo, todo análisis totalizador o de conjunto perderá necesariamente fineza o escrupulosidad y está obligado a situarse a una escala gruesa y poco profunda en términos históricos. De manera similar un análisis puntual digamos de la apropiación o la excreción estará sujeto a una escala más fina y podrá quizás hacer análisis históricos detallados. Todo ello dependiendo, en fin, ya no 
FIGURA 5. El análisis del metabolismo social se torna tridimensional porque puede ser abordado por el investigador en función de la escala espacial, la temporalidad y la dimensión (el o los procesos metabólicos analizados). Aquí se ilustran cinco ejemplos hipotéticos, cada uno en una posición diferente con respecto a los tres aspectos anteriores. Véase texto.

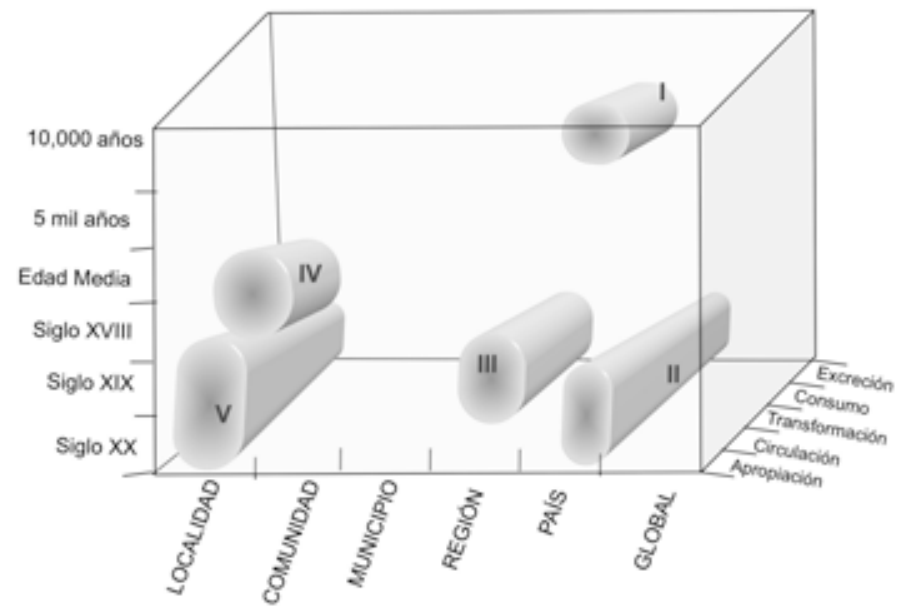

Fuente: González de Molina y Toledo, 2011.

de la destreza del analista sino de la disponibilidad de evidencias, datos y fuentes de información.

\section{LAS TRES FORMAS BÁSICAS DE APROPIACIÓN}

Durante la apropiación, los seres humanos realizan tres tipos básicos de intervención en los espacios naturales, cada uno de los cuales impacta o afecta de manera diferente los ecosistemas y paisajes que son objeto de la apropiación, y cada uno de los cuales termina teniendo una expresión territorial o espacial. En el primer caso, la apropiación se realiza sin provocar cambios sustanciales en la estructura, arquitectura, dinámica y evolución de los ecosistemas y paisajes que se apropian. Aquí se incluyen todas las formas conocidas de caza, pesca, recolección y pastoreo, así como ciertas formas de extracción y de ganadería por forrajeo en las vegetaciones originales. 
En el segundo caso se trata de actos de apropiación donde la acción humana desarticula o desorganiza los ecosistemas que se apropia, para introducir conjuntos de especies domesticadas o en proceso de domesticación, tal y como sucede con todas las formas de agricultura, ganadería, forestería de plantaciones y acuacultura. La principal diferencia entre estas dos modalidades de apropiación de la naturaleza radica en que mientras en el primer caso los ecosistemas se apropian sin afectar su capacidad intrínseca o natural de automantenerse, autorrepararse y autorreproducirse, en el segundo los ecosistemas apropiados han perdido tales habilidades y requieren a fortiori de energía externa (humana, animal o fósil) para mantenerse. En ausencia de la acción humana estos ecosistemas o bien se regeneran y retornan mediante los mecanismos de restauración ecológica a las formas originales de las cuales surgieron, o bien derivan en formas extrańas, atípicas e impredecibles. En el primer caso se trata de una "naturaleza intervenida", en el segundo de una "naturaleza domesticada".

En las últimas décadas la acción conservacionista, que busca la preservación o protección de áreas naturales intocadas o en proceso de regeneración, ha dado lugar a una tercera forma de apropiación en la que los ecosistemas se conservan con fines de protección de especies, patrones y procesos, cuyo mantenimiento resulta de utilidad porque genera servicios tales como el mantenimiento de la diversidad biológica y genética y del clima local, regional o global, la captación de agua, la captura de carbono, el esparcimiento, la educación, la contemplación estética y la investigación científica. Este tercer caso se distingue por ser la acción humana una suerte de "no acción", en el que se suprime todo acto de extracción de bienes del objeto de la apropiación, al cual se busca preservar o proteger por su valor como suministrador de servicios.

Estas tres formas básicas de apropiación de la naturaleza tienen siempre una expresión en el espacio y, en consecuencia, es posible distinguir tres tipos bien demarcados de paisajes o megaambientes: el Medio Ambiente Utilizado (MAU), el Medio Ambiente Transformado (MAT) y el Medio Ambiente Conservado (MAC), así como "formas" de transición o paisajes intermedios entre ellos. A estas tres 
Figura 6. Los flujos de materia, energía, servicios y monetarios que se establecen entre cinco instancias, representadas por cinco pisos (de abajo hacia arriba): el medio ambiente conservado (MAC), el medio ambiente utilizado (MAU), el medio ambiente transformado (MAT), el sector rural y el sector urbano-industrial.

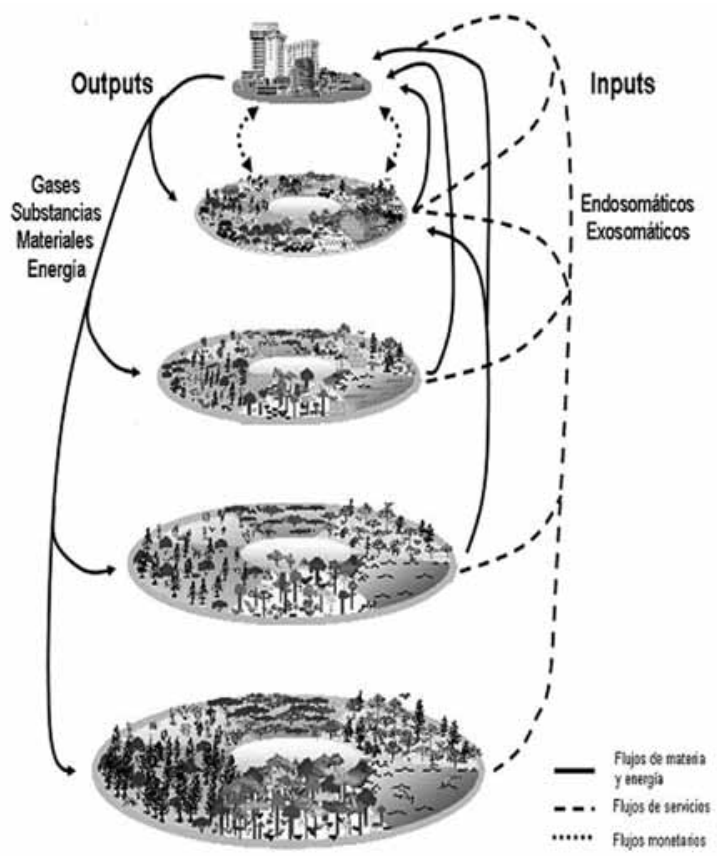

Fuente: González de Molina y Toledo, 2011.

dimensiones "naturales", que son los tres espacios de los que toda unidad de apropiación (P) obtiene flujos de materia, energía y/o servicios, es decir, con los cuales se realizan los intercambios ecológicos, se debe agregar una cuarta, el Medio Ambiente Social (MAS), que son todos aquellos sectores con los cuales toda P realiza los intercambios económicos. Todo ello a su vez permite visualizar el flujo metabólico material, energético, monetario y de servicios, entre los tres medios ambientes y dos sectores sociales: el rural y el urbano (e) industrial (figura 6). El abordaje integrado de los intercambios ecológicos y los intercambios económicos se hace entonces factible mediante un 
modelo básico centrado en el acto de apropiación (véase Toledo 1981, 1990 y 2008; Gonzalez de Molina y Toledo 2011, cap. 2).

\section{LAS ESCALAS DEL METABOLISMO RURAL O AGRARIO}

Toda apropiación tiene lugar dentro de una matriz territorial o espacial, la cual contiene varias escalas, lo que hace que los procesos que se estudian se articulen de manera jerárquica. El análisis del proceso de apropiación puede entonces realizarse en diferentes escalas, cada una de las cuales se encuentra determinada por y determina a las otras escalas. Las escalas en las que puede analizarse el proceso de apropiación van desde aquella marcada por el examen de una sola unidad de apropiación (P) (matizada por una variación determinada por el tamaño de la propiedad de P) hasta todas las $\mathrm{P}$ del planeta, es decir, desde lo "local" hasta lo "global". Entre estas dos situaciones extremas existe toda una gama de situaciones definidas por la amplitud de la escala.

En realidad, siguiendo a Zonneveld (1995), en la amplitud de este espectro pueden reconocerse tres principales niveles o escalas, cada una responde a diferentes heterogeneidades espaciales. Una primera, conocida como topológica, es identificable desde unos cuantos metros cuadrados hasta varios kilómetros cuadrados (mapas hasta $1: 25,000)$ y refleja esencialmente la heterogeneidad vertical (estratos de vegetación, de los suelos, etcétera). Esta escala es la que ha sido privilegiada por los estudiosos de los ecosistemas. Una segunda, llamada corológica, revela la heterogeneidad horizontal que resulta del análisis de conjuntos o mosaicos de unidades topológicas e implica mapas de entre 1: 100,000 hasta 1: 1,000,000 y es equivalente en un nivel regional o de cuenca hidrológica. Finalmente, la escala geósferica está referida a procesos globales y se expresa en mapas de varias decenas de millones.

El modelo de flujos que hemos definido y descrito permite analizar el proceso de apropiación al nivel de la primera y segunda escalas (rango determinado por el tamańo de la propiedad que contiene los ecosistemas/paisajes que P se apropia), y este análisis puede irse amplificando gradualmente conforme se van agregando otras uni- 
Figura 7. Un ejemplo hipotético de análisis multiescalar aparece cuando se determinan los intercambios económicos que varias unidades $\mathrm{P}$ (hogares) realizan dentro de las comunidades a las que pertenecen $(\mathrm{C} 1$ a C4), una cooperativa y/o los mercados regionales, nacional y global.

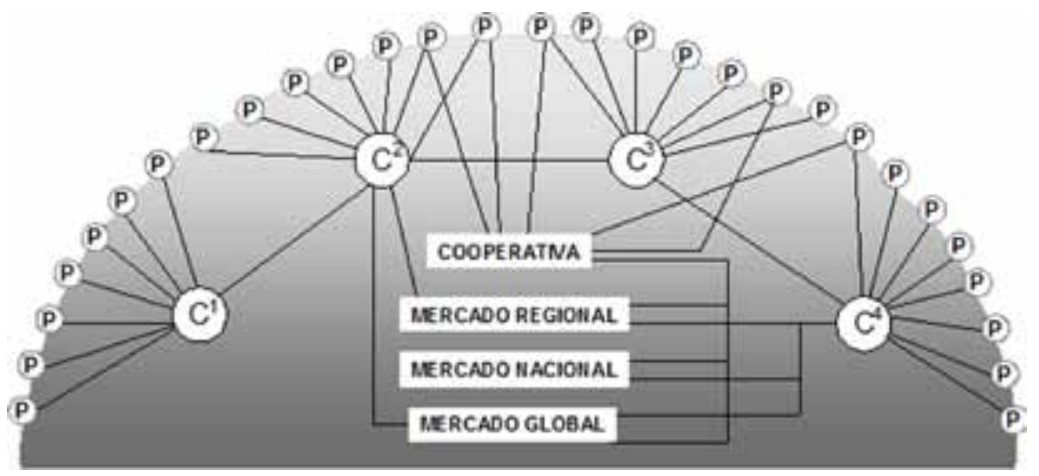

Fuente: González de Molina y Toledo, 2011.

dades $\mathrm{P}$ al campo de dicho análisis. La articulación de varias $\mathrm{P}$ de un cierto territorio, por ejemplo, un conjunto de hogares pertenecientes a una comunidad rural, permite pasar a un siguiente nivel, y la conexión, siempre a través de los intercambios económicos, de varias comunidades rurales en un municipio o región permitiría realizar un análisis de escala regional (figura 7).

En realidad conforme el analista se mueve en la escala, es decir, por los diferentes niveles de agregación de las unidades P, entre ésas y con el MAs, van develándose diferentes configuraciones del metabolismo en los territorios concretos y, al mismo tiempo, se va descubriendo toda una variedad de articulaciones ecológicas y económicas y las sinergias por ellas desencadenadas. También van revelándose las causas y los efectos que los procesos de diferentes escalas van provocando en dos sentidos: hacia las escalas mayores y hacia las escalas menores.

Debe además señalarse que en cada una de las escalas es posible realizar una representación cartográfica del proceso de apropiación, la cual a su vez puede tener una perspectiva histórica (expresión diacrónica del espacio) (véanse ejemplos en González de Molina y Guz- 
mán, 2006; Olarieta et al., 2006 y Marull et al., 2006). De esta forma el modelo de flujos que se postula a una escala específica, se va amplificando y enriqueciendo conforme se va ensanchando la mirada del investigador por los contextos territoriales y sus dinámicas a través del tiempo. Ello significa el ensanchamiento de los espacios geográficos y sociales dentro de los cuales se analizan los metabolismos agrarios, sus procesos y sus articulaciones y sinergias (figura 7).

\section{LAS REGIONES: EL ESTUDIO DE LA TRAMA METABÓLICA}

El incremento de la escala, digamos de una región que incluye tanto comunidades o asentamientos rurales, núcleos urbanos y centros industriales, no sólo permite extender espacialmente el análisis de la apropiación sino, lo que es más importante, permite develar la trama metabólica, esto es, realizar el estudio completo de toda la cadena metabólica en un territorio limitado o particular (por ejemplo, una cuenca hidrológica o una región económica). Ello significa el estudio integrado de los metabolismos agrarios, urbanos e industriales en un espacio regional, el cual es determinado por uno o varios criterios.

A esta escala, el estudio del metabolismo social permite comprender las sinergias y dinámicas que se establecen entre los procesos particulares de apropiación, circulación, transformación, consumo y excreción. El análisis supone la identificación ya no sólo de las unidades de apropiación P, sino de las unidades que se dedican a circular y transformar lo apropiado y/o producido por $\mathrm{P}$, y de los núcleos de consumidores rurales, urbanos e industriales que consumen lo producido, circulado y transformado. Finalmente, el análisis también puede detectar los flujos que terminan siendo excretados hacia la naturaleza por los anteriores procesos en forma de residuos, basura, substancias y emisiones.

El estudio de la trama metabólica supone entonces la identificación de los flujos materiales a través de toda la cadena y la exploración de las dinámicas que se gestan entre las unidades que manejan esos flujos. Como puede suponerse, a esta escala regional el nivel de los fenómenos alcanza tal complejidad que implica un grado alto de dificultad para los investigadores. Ello obliga o bien a incrementar el 
número, la variedad disciplinaria y la calidad de los investigadores, o bien a enfocar el análisis a uno o unos pocos productos del flujo total, por ejemplo agua, combustibles, alimentos, materias primas, etcétera.

El estudio de la trama metabólica en escala regional, está llamado a constituir una unidad de análisis de enorme valor, cuya agregación permite entender dinámicas metabólicas territoriales de diferentes escalas, y descomponer sinergias macro o multirregionales que por su muy alta complejidad resultan incomprensibles o inaccesibles a la investigación. La concatenación de los metabolismos regionales va, a su vez, develando nuevos fenómenos cuyo siguiente límite está marcado por las fronteras nacionales.

\section{EL ESTUdio DEL METABOLISMO A ESCALA NACIONAL}

En última instancia todo estado-nación $(\mathrm{N})$ opera, en términos de intercambios y flujos materiales, como lo hace toda unidad de apropiación/producción $(\mathrm{P})$, es decir, reproduce a una escala infinitamente mayor la misma situación de P. En efecto, toda unidad de escala nacional $\mathrm{N}$ obtiene de su "extracción doméstica", es decir, de los recursos embebidos en su territorio un cierto flujo de entrada (input), el cual es divisible en tres flujos dependiendo del origen de lo que se apropia y produce, pues todo territorio nacional está finalmente integrado por recursos de un Medio Ambiente Utilizado (MAU), un Medio Ambiente Transformado (MAT) y un Medio Ambiente Conservado (MAC). Este flujo de entrada es el resultado de la suma de todos y cada uno de los flujos obtenidos por el conjunto de P pertenecientes a la nación $(\mathrm{N})$.

A la escala nacional, el estudio del metabolismo integra un nuevo flujo. Puesto que dentro de las "entrańas" de un país los flujos de bienes y servicios, resultado de la apropiación del conjunto de unidades $\mathrm{P}$ que habitan el territorio nacional, circulan, se transforman de innumerables formas y son consumidos a distancia por un número inconmensurable de individuos y sectores, aparece un nuevo proceso que permanecía o inidentificable o reducido a su mínima expresión en la escala de P: la excreción. Y es que la trama metabólica 
Figura 8. Esquema del metabolismo de un estado-nación, el cual depende de los intercambios realizados con los recursos de su propio territorio (inputs y outputs), y de los intercambios con otros estadosnación (importaciones y exportaciones).

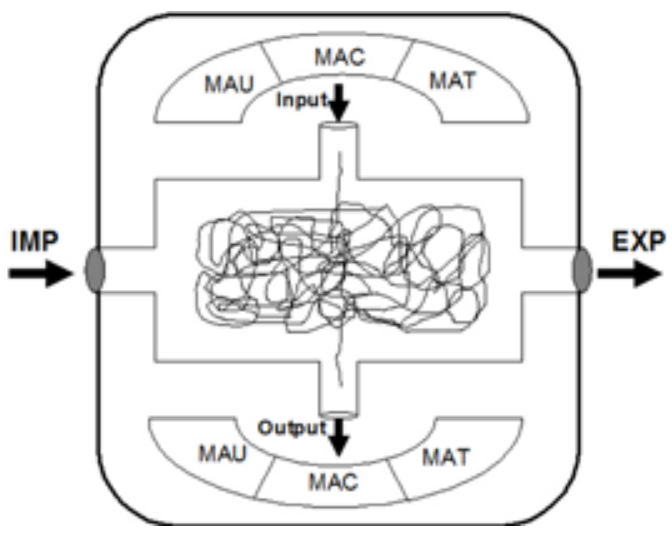

Fuente: González de Molina y Toledo, 2011.

no sólo genera, en cada segmento, un flujo de desechos que terminan en los circuitos del universo natural, sino que dado lo anterior acaba cerrando el proceso general de metabolismo entre la sociedad y la naturaleza al arrojar o excretar agua, materiales y energías que deben ser absorbidas (es decir recicladas) por los ecosistemas.

Los mismos tres ambientes reciben todos los flujos excretados por $\mathrm{N}$, los cuales en conjunto conforman el flujo de salida (output). En teoría deberían poder definirse unidades de excreción E; sin embargo, esta tarea es prácticamente imposible por la enorme variedad de fuentes de expulsión de desechos.

Dado que no existe país que se mantenga reproduciendo su metabolismo sin ningún intercambio más allá de su fronteras, a los dos flujos anteriores se deben sumar los que se establecen con el resto del mundo, es decir, con otros estados-naciones. Por ello, toda nación $(\mathrm{N})$ recibe mediante el intercambio comercial bienes y servicios del resto del mundo (los otros países) los cuales intercambia por productos y bienes generados dentro de su propio territorio (importación y exportación) (figura 8). 


\section{El metabolismo a ESCALA GLOBAL}

El intercambio de flujos de materia y energía entre países, da lugar a un enjambre de relaciones, que en conjunto representan el metabolismo de escala global. La situación particular de cada país, con diferentes grados de complejidad social, cultural, tecnológica, informática, etcétera, junto con el tamańo de los territorios nacionales y la calidad y cantidad de recursos embebidos en ellos, así como su variedad de ecosistemas y paisajes, hace que los intercambios metabólicos adquieran connotaciones diversas y, que por consecuencia, se gesten nuevas situaciones no visualizadas en las otras escalas. Por ejemplo el intercambio desigual y la consecuente importación/exportación de orden (exergía) y desorden (entropía) entre conjuntos de países dentro del Sistema-Mundo (véase Hornborg 2007; Frank 2007; Wallerstein 2007 ), los flujos globales de materia y energía (Naredo 2006), o los impactos producidos y expresados bajo el concepto de la huella ecológica (Wackernagel y Rees 1996).

Figura 9. Las conexiones metabólicas entre los países $(\mathrm{N})$ y entre cada uno de ellos y sus propios recursos naturales constituyen el objeto de estudio del metabolismo internacional.

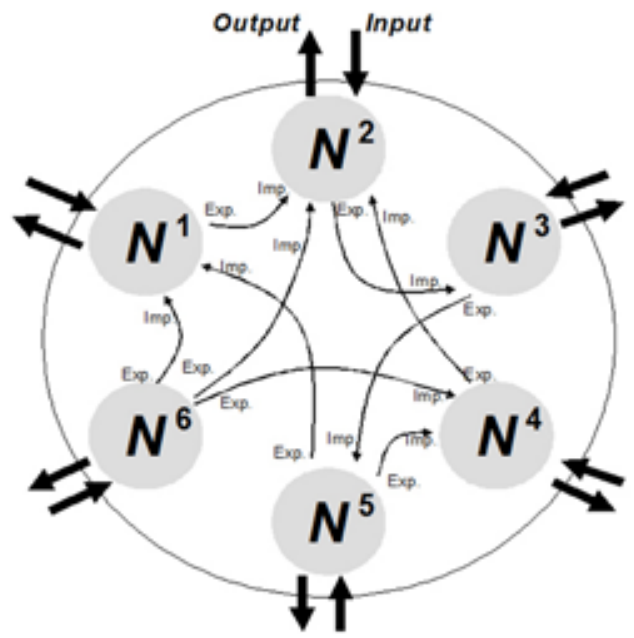

Fuente: González de Molina y Toledo, 2011. 
Este nivel también ha comenzado a ser analizado no solamente al comparar perfiles metabólicos por país, sino cuando por ejemplo se estudian transformaciones de los flujos de materia y energía en regiones, es decir, en conjuntos de naciones pertenecientes a un cierto territorio (e.g. Schandl et al., 2008).

\section{FINAL}

Aunque no existe consenso sobre su definición teórica, el concepto de metabolismo social ha ganado creciente audiencia en los medios académicos, principalmente entre las corrientes de la economía ambiental y ecológica, la historia ambiental y la ecología política. Igualmente será un concepto clave en la construcción de una economía ecológica radical y/o solidaria (véase Barkin et al., 2012 y RosasBańos, 2012). También ha logrado, indirectamente, una primera e interesante convergencia entre la Teoría del Sistema Mundo de E. Wallerstein y los estudiosos del cambio climático global y la destrucción ecológica (Wallerstein 2007). La última década rebosa de publicaciones sobre el tema, incluyendo algunos libros (Fisher-Kowalski y Husserl 2007; Giampietro et al., 2012; González de Molina y Toledo 2011).

El panorama actual deja entrever núcleos de investigadores e instituciones realizando estudios interdisciplinarios a partir del concepto aunque bajo diferentes interpretaciones y con diferentes fines. El primero es el Grupo de Viena representado por el Social Ecology Institute fundado por Marina Fisher-Kowalski. Este grupo, pionero, ha generado abundantes publicaciones en los últimos años y ha introducido nuevos conceptos que ha aplicado en estudios empíricos, tales como el de regímenes sociometabólicos y el de transición sociometábolica (e.g. Kraussman et al., 2008; Haberl et al., 2011). También ha creado y desarrollado una metodología conocida como MEFA (siglas en inglés de análisis de flujos de energía y materia). Su enfoque se concentra en la dimensión material o tangible del metabolismo social. Otros dos grupos tienen como sede la ciudad de Barcelona, España, y dos instituciones. En la Universidad Autónoma de Barcelona, J. Martinez-Alier ha creado un núcleo de investi- 
gadores con numerosos estudiantes que realizan investigación sobre el tema, con autores como M. Giampietro y J.Ramos-Martin. Por su parte E. Tello ha liderado estudios con un interesante enfoque espacial e histórico en la Universidad de Barcelona (Facultad de Economía), en colaboración con historiadores como R. Garrabou y varios geógrafos, quienes se han dedicado a realizar análisis territoriales del metabolismos social en perspectiva histórica.

Un cuarto grupo existe también en España en el Laboratorio de Historia de los Agroecosistemas de la Universidad Pablo de Olavide en Sevilla, bajo la dirección del historiador M. González de Molina, quienes han generado numerosas publicaciones teóricas y de estudios de caso. Finalmente sin ser su tema central ni único, este autor ha impulsado, con algunos colegas y alumnos, investigación teórica y aplicada utilizando el concepto. Dos líneas han sido desarrolladas, ambas en la dimensión rural: la primera a la escala de comunidades (véase Toledo 2008; Cordón y Toledo 2008; García-Frapolli et al., 2008); y la segunda sobre la transición del metabolismo orgánico, agrario o campesino al metabolismo industrial, para lo cual se diseñó una tipología de productores rurales que ha sido aplicada a escala nacional, estatal y en numerosas comunidades (Toledo et al., 2003).

\section{REFERENCIAS}

Ayres, Robert U. y Udo Ernst Simonis, eds., Industrial Metabolism: Restructuring for Sustainable Development, Nueva York, UNU Press, 1994.

Barkin, David, Mario E. Fuente-Carrasco y Daniel Tagle-ZAMORA, "La significación de una Economía Ecológica radical", Revista Iberoamericana de Economía Ecológica 19, 2012, 1-14.

CARPINTERo, Óscar, El Metabolismo de la Economía Española: recursos naturales y huella ecológica (1955-2000), Lanzarote, Fundación César Manrique, 2005.

Colp, Ralph, "The contacts between Karl Marx and Charles Darwin", Journal of History of Ideas 35, 1974, 329-336.

Cordón, María Rosa y Víctor Manuel Toledo, "La importancia conservacionista de las comunidades indígenas de Bosawás, Ni- 
caragua: un modelo de flujos", Revista Iberoamericana de Economía Ecológica 7, 2008, 43-60.

EISENMENGER, Nina y Stefan Giljum, "Evidence from societal metabolism studies for ecological unequal trade", en Alf Hornborg y Carole Crumley, eds., The World System and the Earth System. Global Socioenvironmental Change and Sustainability since the Neolithic, Walnut Creek, Left Coast Press, 2007, 288-302.

Fischer-Kowalski, Marina, "Society's metabolism: on the childhood and adolescence of a rising conceptual star, en Michael Redclift y Graham Woodgate, eds., The International Handbook of Environmental Sociology, Cheltenham, Edward Elgar, 1997, 119-137.

, "Society's metabolism: the intellectual history of materials flow analysis, part I, 1860-1970", Journal of Industrial Ecology 2, 1998, 61-77.

Fischer-KowAlski, Marina y Walter HütTler, “Society's metabolism: the intellectual history of materials flow analysis, part II, 1970-1998”, Journal of Industrial Ecology 2, 1999, 107-129.

Fischer-KowalsKi, Marina y Helga WeIsz, "Society as a hybrid between material and symbolic realms", Advances of Human Ecology 27, 2004, 215-251.

Fischer-Kowalski, Marina y Helmut Haberl, eds., Socioecological Transitions and Global Change. Trajectories of Social Metabolism and Land Use, Edward Elgar, 2007, 263 p.

Foster, John Bellamy, Marxismo y ecología, Madrid, El Viejo Topo, 2000.

Frank, Andre Gunder, "Entropy generation and displacement: the Nineteenth-century multilateral network of World Trade", en Alf Hornborg y Carole Crumley, eds., The World System and the Earth System, Walnut Creek, Left Coast Press, 2007, 303-316.

Gandler, Stefan, "Alfred Schmidt", La Jornada Semanal 916, 2012, 16.

García-Frapolli, Eduardo, Joan Martinez-Alier y Víctor Manuel Toledo, "Apropiación de la naturaleza por una comunidad maya yucateca", Revista Iberoamericana de Economía Ecológica 7 , 2008a, 27-42. 
García-Frapolli, Eduardo, Víctor Manuel Toledo y Joan MartiNEZ-ALIER, "Adaptations of a Yucatec Maya multiple-use ecological management strategy to ecotourism", Ecology and Society 13(2), 2008b, 31, en línea, URL: http://www.ecologyandsociety. org/vol13/iss $2 /$ art31/.*

Gerratana, Valentino, "Marx and Darwin", New Left Review 82, 1973, 60-82. http://www.speciesoforigin.org/downloads/Gerratana_Marx_and_Darwin.pdf

Giampietro, Mario, Kozo Mayuma y Alevgül H. Sorman, The Metabolic Pattern of Society, Routledge, 2012, 405 p.

GonzÁlez de Molina, Manuel, "A guide to studying the socioecological transition in European agriculture", Sociedad Española de Historia Agraria, Documento de Trabajo DT-SEHA, núm. 10-06, www.seha.info, 2010.

GonzÁlez de Molina, Manuel y Gloria I. Guzmán-Casado, Tras los Pasos de la Insustentabilidad: agricultura y medio ambiente en perspectiva histórica, Barcelona, Icaria editorial, 2006, 502 p.

GonzÁlez de Molina, Manuel y Víctor Manuel Toledo, Metabolismos, naturaleza e historia: hacia una teoría socio-ecológica de las transformaciones, Barcelona, Icaria editorial, 2011.

Guzmán Casado, Gloria I. y Manuel González de Molina, "Preindustrial agriculture versus organic agriculture. The land cost of sustainability", Land Use Policy, 2008.

Grau-Satorras, Mar, "Ecological knowledge and use of natural resources, are they related? A study case among tribal communities in Kodagu district (Karnataka, India)", Bsc "Environmental Sciences”, Universitat Autónoma de Barcelona, 2010, http:// ddd.uab.cat/pub/trerecpro/2010/hdl_2072_94000/PFC_MarGrauSatorras.txt

Haberl, Helmut, Marina Fisher-Kowalski, Fridolin Krausmann, Joan Martinez-Alier y Verena Winiwater, "A sociometabolic transition towards sustainability?", Sustainable Development 19, 2011, 1-14.

Hornborg Alf y Carole L. Crumley, eds., The World System and the Earth System. Global Socioenvironmental Change and Sustainability since the Neolithic, Walnut Creek, Left Coast Press, 2006. 
KrausmanN, Fridolin, "Land use and industrial modernization: an empirical analysis of human influence on the functioning of ecosystems in Austria 1830-1995", Land Use Policy 18, 2001, 17-26.

"Milk, Manure and muscle power: livestock and transformation of pre-industrial agriculture in Central Europe", Human Ecology 32(6), 2004, 735-772.

Krausmann, Fridolin y Helmut Haberl, "The process of industrialization from the perspective of energetic metabolism: socioeconomic energy flows in Austria 1830-1995", Ecological Economics 41, 2002, 177-201.

Krausmann, Fridolin et al., "Global patterns of socioeconomic biomasss flows in the year 2000: A comprehensive assessment of supply, consumption and constraints", Ecological Economics 65, 2008a, 471-487.

Krausmann, Fridolin, Heinz Schandl y Rolf Peter Sieferle, "Socioecological regime transition in Austria and United Kingdom”, Ecologial Economics 65, 2008b, 187-201.

Lefebvre, Henri, "Marx et la Nature”, La Pensée 198, 1978, 51-62.

McNeILl, John Robert, Something New under the Sun: An environmental history of the Twentieth Century World, Penguin Books, 2000.

Martinez-Alier, Joan, Economía ecológica, México, Fondo de Cultura Económica, 1987.

,"Marx, energy and social metabolism", Encyclopedia of Energy 3, 2004, 825-834.

, "Marxism, Social Metabolism, and Internacional Trade", en Alf Hornborg, John Robert Mcneill y Joan Martínez-Alier, eds., Rethinking Environmental History. World-System History and Global Environmental Change, Lanhan, Altamira Press, 2007, 221-237.

Marull, Joan, Joan Pino, Enric Tello y Josep Maria Mallarach, "Análisis estructural y funcional de la transformación del paisaje agrario en el Vallés durante los últimos 150 años", Areas (Revista Internacional de Ciencias Sociales) 25, 2006, 105-126.

Marx, Karl, Formas que preceden a la producción capitalista, Córdoba, Argentina, Cuadernos del Pasado y Presente, 1971, 74. 
,El capital, vol. 1, tomo 1, México, Siglo XXI Editores, 1975.

MatThews, Emily et al., "The Weight of Nations: material outflows from industrial economies", Washington, World Resources Institute, 2000.

Moscovici, Serge, "Le marxisme et la question naturelle", L'Homme et la Societé 13, 1969, 59-109.

Muradian, Roldan, Martin O'Connor, Joan Martinez-Alier, "Embodied pollution in trade: estimating the environmental load displacement of industrialized countries", Ecological Economics 36, 2002, 281-297.

Muradian, Roldan y Stefan Giljum, "Physical trade flows of pollution-intensive products: historical trend in Europe and the World", en Alf Hornborg et al., eds., Rethinking Environmntal History, Altamira Press, 2007, 307-326.

NAREDo, José Manuel, "El metabolismo de la sociedad industrial y su incidencia planetaria", en José Manuel Naredo y Fernando Parra, eds., Economía, ecología y sostenibilidad en la sociedad actual, Madrid, Siglo XXI Editores, 2000, 193-229.

Olarieta, José Ramón, Fernando Luis RodríGuez-Valle y Enric TelLo, "Conservando y destruyendo suelos, transformando paisajes (el Vallés, Cataluña, 1853-2004)”. Áreas 25, 2006, 39-66.

Opschoor, Johannes Baptist, "Industrial metabolism, economic growth and institutional change", en Michael Redclift y Graham Woodgate, eds., The International Handbook of Environmental Sociology, Cheltenham, Edward Elgar, 1997, 274-286.

Ortiz-Ávila, Tamara y Omar Raúl Masera, "Subsidios y estrategias de producción campesina: el caso de Casas Blancas, México", Revista Iberoamericana de Economía Ecológica 7, 2008, 61-80.

Parsons, Howard L., Marx and Engels on Ecology, Greenwood Press, 1977.

RedClift, Michael y Graham Woodgate, eds., The International Handbook of Environmental Sociology, Cheltenham, Edward Elgar, 1997.

Rosas-Baños, Mara, "Economía Ecológica y Solidaria: rumbo a 
una propuesta teórica integrada que visualice las rutas hacia la transición”, Revista Iberoamericana de Economía Ecológica 18, 2012, 89-103.

Russi, Daniela, Aana Citlalic González, José C. Silva-Macher et al., "Material flow accounting in Chile, Ecuador, Mexico and Peru (1980-2000)”, mecanoscrito, ICTA, Universidad Autónoma de Barcelona, 2006.

Schandl, Heinz, Marina Fisher-Kowalski, Clemens Grunbuhel y Fridolin Krausmann, "Socio-metabolic transitions in developing Asia”, Technological Forecasting and Social Change, 2008.

Schmidt, Alfred, El concepto de naturaleza en Marx, México, Siglo xxi Editores, 1976.

Singh, Simron Jit, Clemens M. Grunbuhel, Heinz Schandl y Niels Schulz, "Social metabolism and labour in a local context: changing environmental relations on Trinket Island", Population and Environment 23, 2001, 71-103.

SkirbeKK, Gunnar, "Marxisme et ecologie”, Espirit 440, 1974, 643652.

Tello, Enric, Ramón Garrabou, Xavier Cussó y José Ramón Olarieta, "Cambios de uso del suelo desde el metabolismo social agrario: el Vallès 1853-2004”, Revista Iberoamericana de Economía Ecológica 7, 2008, 97-115.

Toledo, Víctor Manuel, "Intercambio ecológico e intercambio económico", en Enrique Leff, ed., Biosociología y Articulación de las Ciencias, México, unAM, 1981, 114-147.

"The ecological rationality of peasant production", en Miguel Altieri y Susanna Hecht, eds., Agroecology and Small-Farm Development, CrC, Press, 1990, 51-58.

"Metabolismos rurales: hacia una teoría económico-ecológica de la apropiación de la naturaleza", Revista Iberoamericana de Economía Ecológica 7, 2008, 1-26.

"La crisis de civilización es una crisis de las relaciones de la sociedad industrial con los procesos naturales", Papeles 110 , 2011, 171-177. Entrevista por M. Di Donato: http://www. fuhem.es/media/ecosocial/File/Entrevistas/entrevista $\% 20 \mathrm{a} \% 20$ Victor\%20Toledo_b_M.\%20DI\%20DONATO.pdf 
, "Diez tesis sobre la crisis de la modernidad", Polis Revista Latinoamericana 33, Puesto en línea el 19 diciembre 2012, consultado el 19 diciembre 2012, uRL: http://polis.revues.org/8544 Toledo, Víctor Manuel, Pablo Alarcón-Chaires y Lourdes BARÓN, La modernización rural de México: un análisis socio-ecológico, México, semarnap, unam, 2002, $130 \mathrm{p}$.

Wackernagel, Mathis y Williams E. Rees, Our Ecological Footprint, Filadelfia, New Society Publishers, 1996.

WALlERSTEIN, Immanuel, "The Ecology and the Economy: What Is Rational?", en Alf Hornborg, John Robert McNeill y Joan Martínez-Alier, eds., Rethinking Environmental History. WorldSystem History and Global Environmental Change, Lanhan Altamira Press, 2007, 379-389.

Zonneveld, Isaak Samuel, Landscape Ecology, Amsterdam, spB, Academic Publishing, 1995, 199 p.

FECHA DE RECEPCIÓN DEL ARTíCULO: 15 de octubre de 2012 FECHA DE RECEPCIÓN DE LA VERSIÓN FINAL: 3 de julio de 2013 